\title{
EDITORIAL
}

\section{Acerca del estatuto científico de la epidemiología}

$\mathrm{L}$ a mayoría de los médicos e incluso algunos epidemiólogos están seguros de que la epidemiología es una disciplina que consiste en el manejo experto de técnicas útiles para prevenir y controlar enfermedades en el ámbito poblacional. De acuerdo con este punto de vista, la principal labor del epidemiólogo es la de recoger información y usarla para resolver problemas prácticos. ${ }^{1}$ De esta concepción de la epidemiología se derivan, sin duda, la estructura y el funcionamiento de muchos centros dedicados a la investigación epidemiológica y la mayor parte de los programas empleados para su enseñanza. En tales casos, el papel que se concede al aprendizaje y uso de las técnicas (en especial a las de tipo estadístico) es extraordinariamente relevante, en detrimento de una reflexión más profunda sobre el sustento teórico de la epidemiología. Esto provoca que terminen siendo excepcionales los espacios académicos en los que se puede reflexionar con suficiente profundidad sobre las estructuras conceptuales que dan a la epidemiología su especificidad disciplinaria y su naturaleza científica.

Esta creencia sobre el papel exclusivamente pragmático de la epidemiología, sin embargo, es un fenómeno relativamente nuevo, ya que hasta mediados de este siglo parecía no haber dudas sobre la cientificidad de la epidemiología. Como Naomar de Almeida Filho ha señalado, ${ }^{2}$ Frost, Stallybrass, Greenwood y Terris reconocían abiertamente a la epidemiología como una ciencia. Sin embargo, con el desarrollo de las técnicas de recolección y análisis de datos, la idea de que su aplicación no implicaba mayores demandas teóricas resultó en la creencia de que la epidemiología en realidad sólo era un método de abordaje.

No obstante, aunque la epidemiología moderna es una rama relativamente reciente de las disciplinas de la salud y, por lo tanto, tiene objetivos que no son siempre bien comprendidos, desde sus inicios se ha encontrado inmersa en un verdadero fuego cruzado entre propuestas teóricas de todo cuño, de manera que el debate conceptual nunca ha sido un aspecto secundario entre los epidemiólogos. La enconada lucha que, desde el inicio del siglo pasado, se desarrolló entre los contagionistas y los seguidores de la teoría miasmática, por ejemplo, ha continuado durante todo el siglo XX bajo nuevas modalidades. Actualmente, quizá la más evidente de estas contiendas es la que siguen desarrollando los seguidores del biologicismo y sus opositores, aunque por supuesto que no es la única.

El problema de la medición epidemiológica, por ejemplo, ha revivido con el surgimiento de un número cada vez mayor de estudios epidemiológicos que utilizan técnicas cualitativas. Como se sabe, la introducción del "método numérico" del médico francés Pierre C. A. Louis influyó tan notablemente en los forjadores de la epidemiología moderna que, a partir de sus trabajos, comenzó una intensa búsqueda de regularidades matemáticas en los eventos sanitarios. Aunque los hallazgos nunca alcanzaron las expectativas formuladas por Louis, condujeron al uso de la teoría de los grandes números aplicada al análisis de las estadísticas sanitarias y a la invención de conceptos que hoy son fundamentales para la investigación epidemiológica, como los de "tasa estandarizada", "medición añopersona" y "exposición poblacional". ${ }^{3}$ Con el epidemiólogo inglés Major Greenwood el razonamiento estadístico en epidemiología alcanzó tal arraigo que la posibilidad de realizar estudios cualitativos en este campo se sigue cuestionado frecuentemente.

En 1931, Stallybrass ${ }^{4}$ aportó nuevos elementos al debate conceptual, al postular que la triada de "factores primarios de diseminación" (a los que denominó semilla, sembrador y suelo) era el principal fundamento científico de la epidemiología. Estos factores, 
conocidos en los años treinta como "las tres eses de Stallybrass", fueron retomados al final de los cincuenta por los estadunidenses Leavell y Clark, ${ }^{5}$ quienes los introdujeron en el modelo conocido como "historia natural de la enfermedad". La propuesta tuvo tanto éxito que en pocos años toda la investigación epidemiológica se había volcado hacia la búsqueda de los factores que desencadenaban el rompimiento del equilibrio ecológico señalado por estos autores.

En 1975, Carol Buck reanimó la polémica sobre el estatuto científico de la epidemiología con la publicación de su hoy célebre artículo "Popper's philosophy for epidemiologist". ${ }^{6}$ Entre los aportes de esta publicación destaca el reconocimiento del excepcional valor que los epidemiólogos conceden a su método de estudio. De acuerdo con esta autora, el hecho de que la epidemiología otorgue tanta importancia al aspecto lógico (en oposición al puramente técnico) del método, se debe a que en esta disciplina el experimento juega un papel muy limitado y, en consecuencia, deben crearse cuasi-experimentos usando los fenómenos tal como ocurren naturalmente. El reconocimiento de esta característica del método provocó un nuevo interés en el análisis de los fundamentos lógicos del trabajo epidemiológico, aunque en un plano estrictamente metódico. Para Buck, este interés parcial podría ser más creativo si se conociesen mejor los puntos de vista de la filosofía de las ciencias.

En la actualidad, la epidemiología debe enfrentar varios problemas epistemológicos. De ellos, quizá el más importante es el problema de la causalidad, aspecto sobre el que todavía no existe consenso entre los expertos. El abanico de posturas se extiende desde los que proponen generalizar el uso de los postulados de causalidad (sean éstos los de Henle-Koch, los Bradford Hill o los de Evans) hasta los que consideran que la epidemiología debe abandonar el concepto de "causa" y limitarse a dar explicaciones no deterministas de los eventos que investiga. Las críticas al concepto de causa, formuladas por primera vez por David Hume en 1740, probablemente implicarían replantear conceptos tan arraigados en la investigación epidemiológica como los de "causa necesaria", "componente de causas suficientes" o "riesgo atribuible", por ejemplo. Dado que estas críticas son cada vez más aceptadas por las ciencias naturales, es indudable que este tema seguirá siendo uno de los predilectos de la literatura epidemiológica del siglo XXI.

Otro de los problemas filosóficos de la epidemiología contemporánea es el que se refiere a la índole de su objeto de estudio. En este campo, los distintos esfuerzos por determinar la naturaleza de los eventos epidemiológicos también han desembocado en la for- mación de diversas corrientes, que debaten intensamente si este objeto se alcanza con la suma de lo individual, con el análisis poblacional o mediante la investigación de la colectividad social. Como resultado, han proliferado los intentos por desentrañar, cada vez con mayor rigor, las interacciones que se establecen entre la actividad clínica, la estadística y las ciencias sociales. $^{7}$

El último de los aspectos centrales en este peculiar debate alude al estatuto científico del saber epidemiológico. Aunque ya nadie acepta la posibilidad -por cierto, planteada también por Pierre Louis en el siglo XIX- de que los eventos epidemiológicos podrían comportarse siguiendo leyes similares a las que rigen los fenómenos naturales, los aportes de la epidemiología en el terreno de la generación de teorías, modelos y conceptos han dado numerosos frutos. Algunas muestras son, por ejemplo, los conceptos de "historia natural de la enfermedad", "riesgo" y "base poblacional", utilizados con gran éxito en los últimos treinta o cuarenta años. Los modelos de "propagación epidémica", que surgieron de la amalgama entre la teoría epidemiológica y la probabilidad, siguen aportando instrumentos predictivos cada vez más potentes.

El desarrollo conceptual en la epidemiología, como ha sucedido desde el nacimiento de la forma moderna de la disciplina, lejos de detenerse ha seguido ganando terreno. Los mismos conceptos centrales a los que se aludió antes, como los de "historia natural de la enfermedad" y "riesgo", durante la última década han sido puestos en tela de juicio por los críticos de la epidemiología tradicional. La teoría de la transición epidemiológica (que desde su nacimiento proporcionó valiosos elementos para interpretar la dinámica secular de la enfermedad poblacional) también ha sido objeto de profundas reformulaciones teóricas. ${ }^{8} \mathrm{La}$ "hipótesis de la compresión de la morbilidad", por su parte, se encuentra en proceso de contrastación empírica en varios países, aunque ya permite analizar con mejores herramientas conceptuales algunos eventos sanitarios antes poco explorados, como es el caso de las relaciones entre el envejecimiento poblacional y los límites biológicos de la sobrevivencia. Salud Pública de México presenta, en este número, un ensayo que revisa las implicaciones teóricas y prácticas de esta hipótesis, formulada por primera vez en $1980 .^{9}$

Como parece claro, el problema de la cientificidad de la epidemiología y la revaluación permanente de sus bases conceptuales y metodológicas es un asunto crucial para el proceso de maduración histórica e institucional de la disciplina. Sólo de esta forma podrán combinarse el enorme valor de la epidemiología para diseñar actividades preventivas y de control con ese 
otro valor que la hace capaz de proporcionarnos una imagen más científica de la enfermedad. Este es un compromiso que los epidemiólogos no podemos eludir. Lograrlo depende del esfuerzo y la imaginación que empeñemos en ello.

\section{Dr. Sergio López Moreno*}

\section{Referencias}

1. Savitz DA. The alternative to epidemiology theory: whatever works. Epidemiology 1997;8:210-212.
2. Almeida FN . 0 estatuto científico da epidemiología (Editorial). Rev Saude Publica 1991;25:339-340.

3. Lilienfeld AM, Lilienfeld DE. Fundamentos de epidemiología. México, D.F.: Sistemas Técnicos de Edición, 1986.

4. Nájera LE. Criteriología de la investigación epidemiológica. Anales de Medicina Pública 1952;4:415-484.

5. Leavell HR, Clark F. Preventive medicine for the doctor in his community. Citado por: Breilh J. Epidemiología, economía, medicina y política. 3a. edición. México, D.F.: Editorial Fontamara, 1979.

6. Buck C. Popper's philosophy for epidemiologist. Int J Epidemiol 1975;4:159-168.

7. Almeida FN. A clínica e a epidemiologia. Salvador de Bahía: APCEABRASC 0, 1992.

8. Frenk MJ. La salud de la población. Hacia una nueva salud pública. México, D.F.: Fondo de Cultura Económica, 1993.

9. Fries JF.A ging, natural death and the compression of morbidity. $N$ Engl J Med 1980;303:130-135.

\footnotetext{
* Centro de Investigación en Sistemas de Salud, Instituto Nacional de Salud Pública, México.
} 